\title{
Technical challenges regarding the use of formalin-fixed paraffin embedded (FFPE) tissue specimens for the detection of bacterial alterations in colorectal cancer
}

\author{
Suk Yee Lam ${ }^{1 \dagger}$, Athanasia loannou ${ }^{2 \dagger}$, Prokopis Konstanti ${ }^{2}$, Thijmen Visseren ${ }^{1}$, Michail Doukas ${ }^{3}$,
} Maikel Petrus Peppelenbosch ${ }^{1}$, Clara Belzer ${ }^{2 \dagger}$ and Gwenny Manel Fuhler ${ }^{{ }^{*+}}$

\begin{abstract}
Background: Formalin-fixed paraffin embedded (FFPE) tissues may provide an exciting resource to study microbial associations in human disease, but the use of these low biomass specimens remains challenging. We aimed to reduce unintentional bacterial interference in molecular analysis of FFPE tissues and investigated the feasibility of conducting quantitative polymerase chain reaction (qPCR) and 16S rRNA amplicon sequencing using 14 colorectal cancer, 14 normal adjacent and 13 healthy control tissues.

Results: Bacterial contaminants from the laboratory environment and the co-extraction of human DNA can affect bacterial analysis. The application of undiluted template improves bacterial DNA amplification, allowing the detection of specific bacterial markers (Escherichia coli and Faecalibacterium prausnitzii) by qPCR. Nested and non-nested PCRbased 165 rRNA amplicon sequencing approaches were employed, showing that bacterial communities of tissues and paired paraffin controls cluster separately at genus level on weighted Unifrac in both non-nested (R2 $=0.045$; $\operatorname{Pr}(>F)=0.053)$ and nested $(R 2=0.299 ; \operatorname{Pr}(>F)=0.001) P C R$ datasets. Nevertheless, considerable overlap of bacterial genera within tissues was seen with paraffin, DNA extraction negatives (non-nested PCR) or PCR negatives (nested $P(R)$. Following mathematical decontamination, no differences in $\alpha$ - and $\beta$ diversity were found between tumor, normal adjacent and control tissues.
\end{abstract}

Conclusions: Bacterial marker analysis by qPCR seems feasible using non-normalized template, but 16S rRNA amplicon sequencing remains challenging. Critical evaluation of laboratory procedures and incorporation of positive and negative controls for bacterial analysis of FFPE tissues are essential for quality control and to account for bacterial contaminants.

Keywords: DNA contamination, Gastrointestinal microbiome, High-throughput nucleotide sequencing, Formalinfixed paraffin embedded, Low biomass, Colorectal neoplasms

*Correspondence: g.fuhler@erasmusmc.nl

†Suk Yee Lam and Athanasia loannou share first authorship.

${ }^{\dagger}$ Clara Belzer and Gwenny Manel Fuhler share senior authorship.

1 Department of Gastroenterology and Hepatology, Erasmus MC University Medical Center Rotterdam, P.O. Box 2040, 3000 CA Rotterdam, The Netherlands

Full list of author information is available at the end of the article

\section{Background}

The preservation of formalin-fixed paraffin embedded (FFPE) tissue samples in the archives of health institutes has facilitated the study of human disease worldwide. In contrast to prospectively collected fresh and frozen material, FFPE tissue specimens are readily available to 
investigate a variety of health-related issues [1,2]. Pathology archives are also an exciting potential source of information to answer microbe-related health questions. Both bacterial and viral deoxyribonucleic acid (DNA) can be detected in FFPE tissue specimens and have been used to investigate associations between invading pathogens and diseases, for instance to determine the presence of Helicobacter pylori in gastric adenocarcinoma [3] as well as hepatocellular carcinoma [4] and human papilloma virus in cervical cancer [5]. Since innovative technologies have enabled the identification of microbiota and their genomes (microbiome) in the different niches of the human body [6,7], FFPE tissue specimens might serve as an additional source to map these communities. Research questions which require the investigation of specific disease sites, rare diseases or a long follow-up time of patients may in particular benefit from the use of long-term collection and storage of FFPE tissue material. Examples of such studies include the investigation of colorectal cancer (CRC)-specific microbial composition [8] and the exploration of intestinal bacterial communities in neonates with necrotising enterocolitis [9-11].

Nevertheless, the application of FFPE tissues for microbiome analyses is associated with several challenges. First, obtaining sufficient quantities of genomic DNA of good quality remains difficult [12-15]. Neutrally buffered formalin prevents total DNA degradation [2, 14], but DNA cross-linking and fragmentation $[14,16]$ as well as storage time post-fixation $[15,17]$ impair the recovery of nucleic acids. Whereas the amplification of large DNA fragments is considered problematic due to DNA integrity deterioration [12, 14], shorter fragments have been used for molecular analyses [15-19], even in samples archived for over twenty years [20]. Secondly, FFPE tissues have relatively high human genomic DNA content and are considered low bacterial biomass samples. Amplification steps such as nested polymerase chain reaction (PCR) may improve specificity and sensitivity of detected bacteria. Thirdly, microbial contaminants were shown to be present in commonly used reagents and can critically influence microbiome results, especially in low bacterial biomass samples [21-26]. Since bacterial contamination affects both $16 \mathrm{~S}$ ribosomal RNA (rRNA) amplicon sequencing and shotgun metagenomics [25], careful handling of samples is essential during bacterial DNA retrieval and subsequent molecular analysis.

In this study, our aim is to optimise a method to reduce the interference of non-informative microbial contaminants in order to extract biologically relevant information from FFPE tissue specimens. To investigate the feasibility of conducting microbial analyses, we employed a cohort of $41 \mathrm{FFPE}$ specimens to explore microbial associations in CRC using bacterial marker analysis and 16S rRNA amplicon sequencing. We report the difficulties encountered in an effort to optimize the processing of FFPE tissue specimens for future microbial studies.

\section{Results \\ Bacterial and human DNA interference in microbial analyses of FFPE tissue specimens}

Interference from contaminants is a common problem for samples of low microbial biomass. The 16S rRNA amplicon sequencing pilot results showed a predominance of Ralstonia in five out of eight samples (Fig. 1a). Retrospective analysis demonstrated the elution buffer as the contaminating source while other extraction reagents were excluded (Supplementary Fig. S1a). Differences in bacterial DNA detection were observed for the same set of pathology paraffin collections using two separate kits (Supplementary Fig. S1b), indicating that the extent of contamination varies per newly opened kit and its components (Supplementary Fig. S1c), while paraffin itself is not a contaminating source. Nevertheless, bacterial DNA presence was confirmed in the majority of 41 FFPE tissues and was minor in paired paraffin controls (Fig. 1d), with quantification of the bacterial biomass showing significantly higher $16 \mathrm{~S}$ rRNA gene copies numbers in tissues $(n=39)$ compared to matched paraffin samples $(\mathrm{n}=38)$ (Supplementary Fig. S1d). However, human genomic DNA is co-extracted with bacterial DNA and present in higher concentrations in FFPE tissues $(P=0.002)$ (Fig. 1b, e), and therefore normalisation of samples for DNA concentration may hamper bacterial DNA detection (Fig. 1c). Thus, human and microbial contaminants may interfere in microbial analysis of low biomass samples and should be accounted for.

Bacterial marker analysis of FFPE tissue specimens by qPCR The use of non-normalized template allowed the comparison of bacterial markers in CRC $(n=14)$, normal adjacent tissues $(n=12)$ and healthy tissues $(n=13)$. The fold change $\left(2^{-\Delta \Delta C T}\right)$ levels of Escherichia coli (E.coli) were significantly different among groups $(P=0.013)$, in particular CRC compared to healthy controls (Fig. 2a). A minority of tissue samples harboured $p k s$ positive strains, but no differences were detected among groups (Fig. 2b). The levels of Faecalibacterium prausnitzii (F. prausnitzii) differed between tissue types $(P=0.017)$ with post-hoc analysis indicating significant higher levels in healthy controls compared to CRC (Fig. 2c).

\section{Exploring bacterial communities in FFPE tissues, paraffin and controls with two sequencing approaches}

First, we employed the most commonly used sequencing approach, with amplification of the bacterial 16S rRNA hypervariable region V3-V4. A total of 41 FFPE tissue 


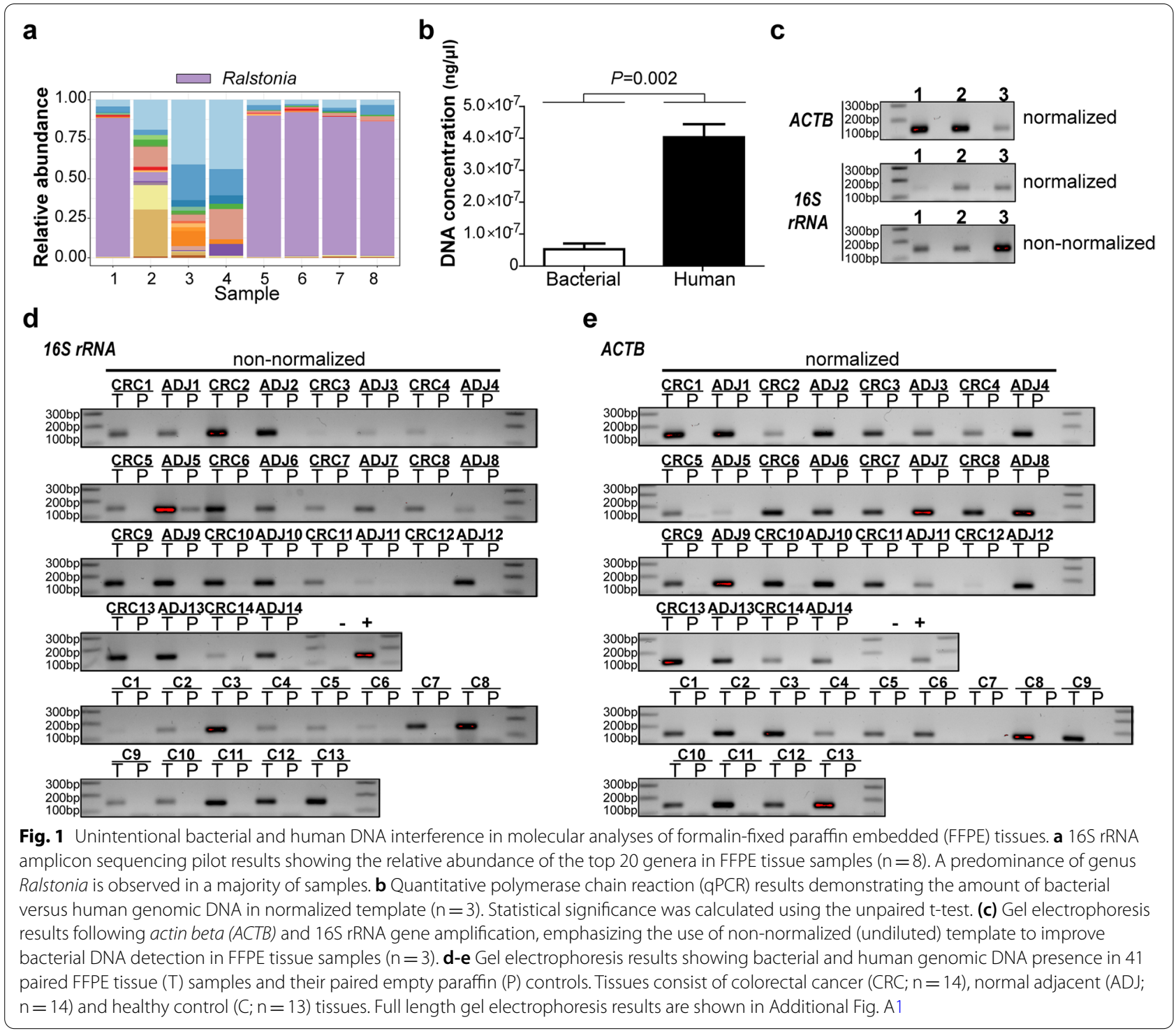

a

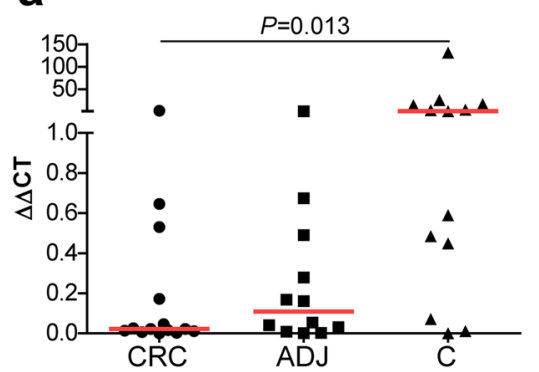

b

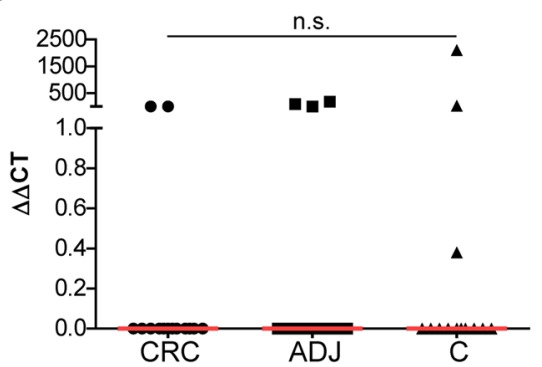

C

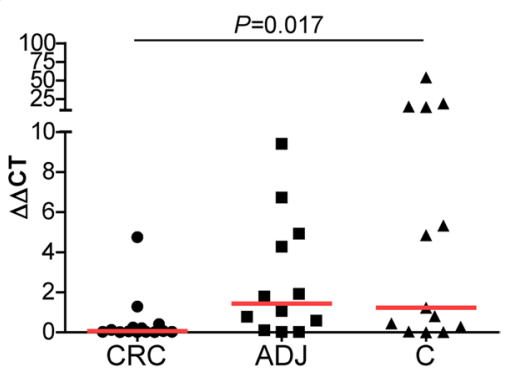

Fig. 2 Quantitative polymerase chain reaction (qPCR) analysis of bacterial markers in formalin-fixed paraffin embedded (FFPE) tissues. GPCR results of specific bacterial markers in colorectal cancer ( $C R C ; n=14)$, normal adjacent ( $A D J ; n=12$ ) and healthy colonic control $(C ; n=13)$ using non-normalized template. The relative amount of each sample is normalized to human genomic DNA and expressed in terms of fold change (2$\Delta \Delta C T$ ). a-b Detection of Escherichia coli (E.coli) (a) and strains harbouring the pks + island (b) using E. coli and ClbA gene specific primers, respectively. c Detection of Faecalibacterium prausnitzii 
samples, six paraffin controls and six DNA extraction negatives were included for sequencing. No differences in Shannon diversity were found at genus level, but the Chao1 diversity index was significantly higher in tissues than DNA extraction negatives $(P<0.010)$ (Fig. $3 a)$. The bacterial communities clustered separately based on weighted UniFrac $(\mathrm{R} 2=0.088 ; \operatorname{Pr}(>\mathrm{F})=0.010)$ and Bray-Curtis dissimilarity $(\mathrm{R} 2=0.116 ; \operatorname{Pr}(>\mathrm{F})=0.001)$, as shown by the Principal Coordinates Analysis (PCoA) plots at genus level (Fig. 3b). Pairwise comparisons indicated that tissues were distinct from DNA extraction negatives on weighted UniFrac $(\mathrm{R} 2=0.063$; $\operatorname{Pr}(>\mathrm{F})=0.017)$ and Bray-Curtis dissimilarity $(\mathrm{R} 2=0.081$; $\operatorname{Pr}(>\mathrm{F})=0.001)$. Tissues also differed from paraffin when computed with Bray-Curtis dissimilarity $(\mathrm{R} 2=0.066$; $\operatorname{Pr}(>\mathrm{F})=0.001)$ while a trend was observed for weighted Unifrac $\quad(\mathrm{R} 2=0.045 ; \quad \operatorname{Pr}(>\mathrm{F})=0.053)$. Homogeneity conditions were met and findings were in agreement at operational taxonomic unit (OTU) level (Supplementary Table S3). The heat map demonstrated a considerable overlap of genera among tissues, paraffin and DNA extraction negatives (Fig. 3c). A total of 53 bacterial families were detected in these latter controls, indicating significant interference from contaminants/artefacts derived during DNA isolation, library preparation and sequencing procedures (Supplementary Fig. S2a).

A second approach using nested PCR for bacterial DNA amplification was performed on 38 FFPE tissues, 21 paraffin controls, two mock communities, six DNA extraction negatives (samples undergoing DNA isolation procedure) and two PCR negatives (samples not undergoing DNA isolation, i.e. PCR/sequencing controls). Shannon and Chao1 diversity indices were higher in tissues than DNA extraction negatives $(P<0.001$ and $P<0.0001)$,

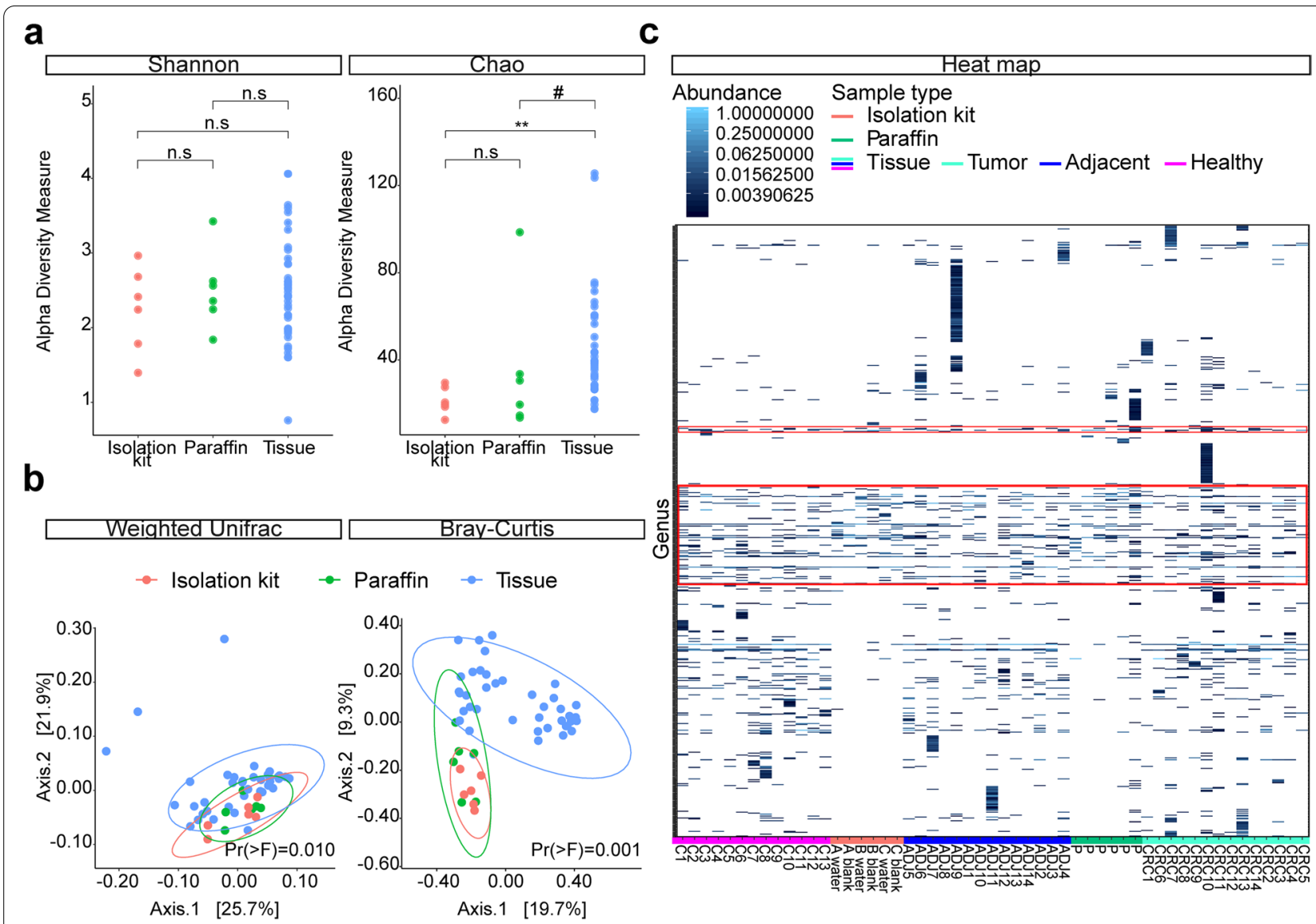

Fig. 3 Targeted 16S rRNA gene amplification and sequencing approach for tissue, paraffin and DNA extraction negatives. The 16S rRNA amplicon sequencing results of formalin-fixed paraffin embedded (FFPE) tissue $(n=41)$, empty paraffin controls $(n=6)$ and DNA extraction controls ( $n=6)$ at genus level using a non-nested polymerase chain reaction (PCR) approach. a Dot plots show the bacterial biodiversity measured by Shannon and Chao1 diversity indices. b Principal Coordinates Analysis (PCoA) plots illustrate the bacterial composition of tissue, paraffin and DNA extraction controls using weighted UniFrac and Bray-Curtis dissimilarity. $\mathbf{c}$ Heat map of the relative abundance of genera across samples. Tissue specimens consist of tumor $(n=14)$, normal adjacent tissue $(n=14)$ and healthy colonic tissue $(n=13)$. The red box indicates genera present in DNA extraction negatives and other samples. Abbreviations for level of significance: $n . s$. not significant; $\#, P<0.1 ; * *, P<0.01$ 
but lower compared to paraffin (both $P<0.0001$ ) (Fig. 4a). No differences in $\alpha$-diversity were observed between tissues and PCR negatives. Moreover, the community structure among the five groups were significantly different based on weighted UniFrac ( $2=0.374 ; \operatorname{Pr}(>F)=0.001$ ) (Fig. 4b). Tissues were distinct from paraffin $(\mathrm{R} 2=0.299$; $\operatorname{Pr}(>\mathrm{F})=0.001)$ and DNA extraction negatives $(\mathrm{R} 2=0.155 ; \operatorname{Pr}(>\mathrm{F})=0.001)$, but not from $\mathrm{PCR}$ negatives $(\mathrm{R} 2=0.055 ; \operatorname{Pr}(>\mathrm{F})=0.056)$. Also paraffin did not differ from these PCR controls ( $R 2=0.061 ; \operatorname{Pr}(>F)=0.234$ ). When using Bray-Curtis dissimilarity for overall group comparison, bacterial communities clustered separately $(\mathrm{R} 2=0.361 ; \operatorname{Pr}(>\mathrm{F})=0.001)$, albeit heterogeneous dispersion $(\operatorname{Pr}(>F)=0.004)$ was noticed (Fig. 4b). Tissues differed from DNA extraction negatives $(\mathrm{R} 2=0.089$;
$\operatorname{Pr}(>\mathrm{F})=0.001) \quad$ and $\quad \mathrm{PCR}$ negatives $\quad(\mathrm{R} 2=0.054$; $\operatorname{Pr}(>F)=0.020)$, but pairwise comparison with paraffin did not meet the condition of homogenous dispersion. No significant differences were found between paraffin and PCR negatives $(R 2=0.072 ; \operatorname{Pr}(>F)=0.219)$. Analyses were also performed at OTU level, which showed similar results (Supplementary Table S3). Multiple bacterial genera were shared between tissues, paraffin and PCR negatives, but not with DNA extraction negatives and mock controls (Fig. 4c). Six bacterial families were found in DNA extraction negatives (Supplementary Fig. S2a). To estimate the accuracy of the experimental procedure and the pipeline, Pearson correlation for theoretical and experimental mock communities was calculated. The correlation was $\mathrm{r}=0.923(P=0.064)$ and $\mathrm{r}=0.952$

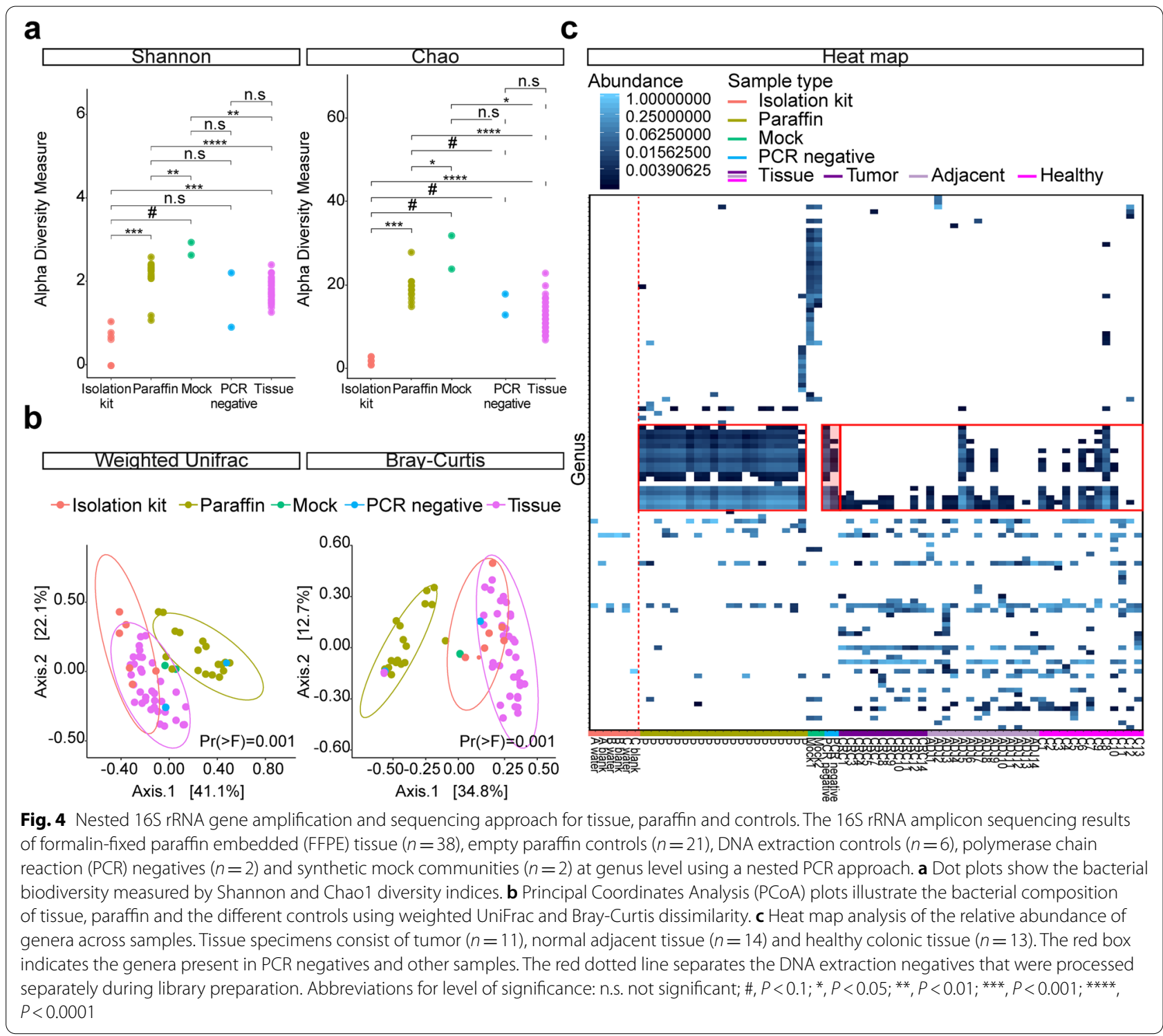


$(P=0.064)$ for the mock controls, respectively (Supplementary Fig. S2b).

\section{Retrieving biologically relevant information from FFPE samples remains challenging}

The library size of both datasets comprised of low read numbers overall for FFPE tissues which overlapped with DNA extraction negatives in the non-nested PCR data set and PCR negatives in the nested PCR dataset (Supplementary Fig. S3a,b). These controls were set for the identification of contaminants using the prevalence method at a threshold of 0.5 that was selected based on its discriminative ability (Supplementary Fig. S3c,d) [27]. This resulted in the removal of 1.006 .017 (13.8\%) and $5.464 .548(22.4 \%)$ reads corresponding to the biopsy samples in respectively the non-nested and nested PCR datasets. The remaining 1684 OTUs within the nonnested PCR dataset following decontamination belonged to 42 phyla including Proteobacteria (30.6\%), Firmicutes
(29.2\%) and Bacteroidetes (14.4\%) while the nested PCR data set comprised of 440 OTUs from six phyla including Proteobacteria (45.2\%), Firmicutes $(41.8 \%)$ and Actinobacteria $(8.2 \%)$ in descending order (Supplementary Fig. S4a,b). Deinococcus-Thermus from both data sets and many spurious others from the non-nested PCR data set (e.g. Planctomycetes) are generally not seen in fecal samples [28], which might hamper the retrieval of biologically relevant information. Nevertheless, the detection of Faecalibacterium and Escherichia-Shigella species within tissue samples was indeed possible by sequencing. After decontamination, there were no differences in $\alpha$ - and $\beta$ diversity between tumor, normal adjacent and healthy tissues in the non-nested dataset at genus level (Fig. 5a). The nested dataset failed to meet the homogeneity condition of permutational multivariate analyses of variance (PERMANOVA), thus rendering dispersion as the possible reason for significant differences in $\beta$-diversity (Fig. 5b).

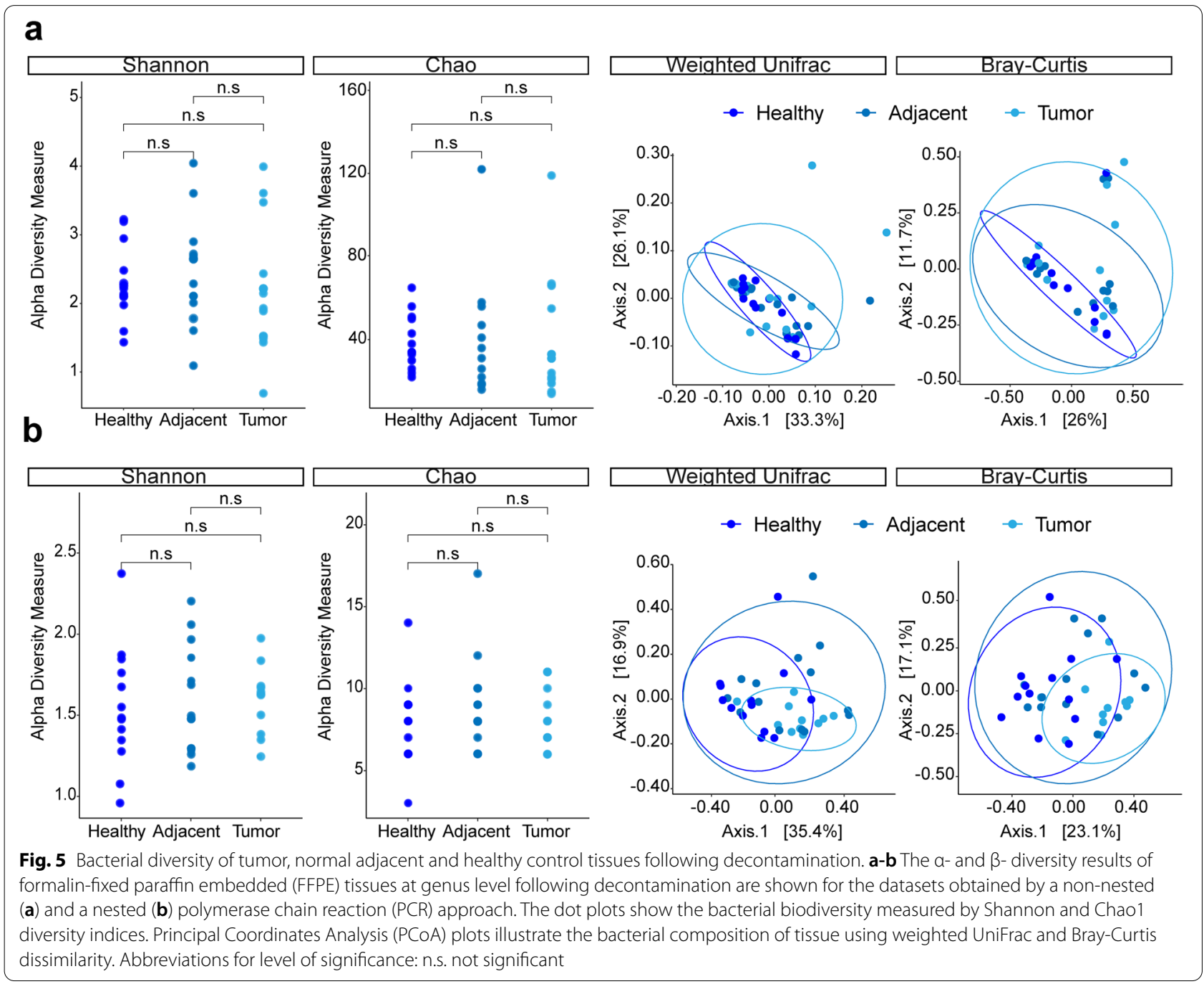




\section{Discussion}

FFPE tissue specimens may provide an important source to study the microbiota in health and disease, but their use is associated with several technical challenges. Our study demonstrates that despite the use of specialised bacterial DNA isolation kits, normalisation of low biomass FFPE tissue samples is primarily driven by human DNA rather than bacterial DNA presence. Our study also shows that the use of non-normalized (undiluted) template may improve bacterial detection in downstream bacterial analyses. Although bacterial marker analysis by qPCR was feasible for two selected markers (Fig. 2), high throughput analyses would require hundreds of individual qPCR assays and sequencing efforts may thus prove to be more efficient. Nevertheless, we show that the extraction of biological relevant information from 16S rRNA amplicon sequencing data remains difficult. Our sequencing pilot was distorted by a previously reported contaminant, Ralstonia [22, 25, 26, 29], underscoring the critical impact of bacterial contamination on low biomass samples as described by others [21-26]. We applied and recommend stringent measures for processing FFPE tissues to reduce the chance of contamination (Supplementary Fig. S5), but it should be noted that PCR negatives not undergoing DNA extraction also showed significant presence of bacterial DNA, which were either introduced during PCR and sequencing efforts, or the result of sequencing artefacts arising as a result of low biomass (Fig. 4c). In particular the detection of biologically relevant taxa such as the Bifidobacteriaceae, Lactobacillaceae and Enterobacteriaceae families in negative controls (Supplementary Fig. S2a) can reduce the robustness concerning the presence of such taxa in samples. Since negative controls demonstrate both the nature and the source of contamination, quality control at different steps of sequencing efforts is particularly recommended. The inclusion of paraffin controls allowed us to demonstrate that bacterial communities of tissues and paraffin tend to cluster separately at genus level in both datasets, and therefore paraffin seems less informative regarding to contaminants. However, the incorporation of DNA extraction negatives and PCR negatives showed that these low biomass samples (tissue and paraffin) were highly affected by bacterial contaminants and/or sequencing artefacts, in line with recent literature $[24,30]$. Synthetic mock controls with high bacterial biomass were less affected, but future studies should consider mock controls with both high and low concentration [31]. Controls with similar microbial biomass as the experimental samples would be representative for the actual effect of contamination and the loss of specific signal due to sequencing efforts.
Knowledge of the characteristics of the investigated ecosystem and the introduction of controls is important for the critical appraisal of results [22]. The DNA quality obtained from FFPE tissue specimens allowed bacterial marker analysis by qPCR. In addition to the detection of E.coli and ClbA gene positive strains, the finding of lower F. prausnitzii levels in CRC compared to healthy controls is in accordance to previous findings $[32,33]$. Our sequencing efforts indeed indicated the presence of Escherichia spp and Faecalibaterium spp in a part of the FFPE tissues. The finding of Proteobacteria as the major phylum in both datasets is not fully understood, but in line with a recent study suggesting that the paraffin embedding process might influence the microbial profile [34]. Multiple aspects in different processes involving pre-processing of tissues, storage conditions, DNA isolation, library preparation and actual sequencing are known to influence the outcome [21, 24-26]. While FFPE tissue specimens have previously been used for $16 \mathrm{~S}$ rRNA amplicon sequencing $[8,10]$ and also for shotgun metagenomics study recently [35], data retrieval is limited compared to frozen tissues [34]. Nevertheless, FFPE samples are sometimes the only available source to answer research questions, allowing complementary taxonomic and functional exploration the microbiome despite relatively low read counts [35]. Thus, when using this material, we highly recommend the identification of prominent contaminating sources to increase the robustness of the dataset's biological information.

Our study has several limitations. First, our bacterial amplification approaches were not directly comparable due to different library preparations and sequencing platforms. Although PCR negatives and mock controls were not available in both sequencing efforts, their findings together emphasized the importance to include both positive and negative controls to account for bacterial contamination. Secondly, the retrieval of biological relevant information from low abundance bacterial DNA of questionable quality and quantity remains challenging. The maximum DNA fragment size detectable was not determined for each of our DNA samples, but it might be helpful to guide the experimental set-up in future studies using FFPE tissues. Although the exploration of alternative tissue fixation and isolation protocols was also out of our scope, bacterial marker analysis by qPCR with FFPE tissue specimens was possible here. Thirdly, the decontamination process leads to loss of both data in general and possibly rare bacterial taxa in low bacterial biomass samples, which should be taken into consideration by researchers when extrapolating such results. Lastly, individual laboratory reagents for library preparation were not tested, but the inclusion PCR negatives are essential to account for procedural related contamination and to 
interpret results. The application of enzymatic treatment of PCR master mixes has been suggested [26], and might be considered in future efforts.

\section{Conclusions}

Our study with FFPE tissue specimens has stressed the importance to implement measures against bacterial contamination in microbiome research with low bacterial biomass samples. Since human genomic DNA is being co-extracted from FFPE tissues, the use of nonnormalized (undiluted) template is recommended for bacterial detection in downstream molecular analyses. While the execution of $16 \mathrm{~S}$ rRNA amplicon sequencing on FFPE tissue specimens remains difficult, the inclusion of negative controls (e.g. DNA extraction negatives and PCR negatives) and positive controls (e.g. synthetic mock communities) is important for quality control, and the use of only one source of contamination control may not be sufficient. Future microbiome studies with low biomass specimens should critically evaluate laboratory procedures to account for bacterial contamination.

\section{Methods}

\section{FFPE tissue and paraffin collections}

FFPE colonic tissue specimens $(n=8)$ were used for our initial 16S rRNA amplicon sequencing pilot. A total of 41 FFPE tissue specimens, containing CRC $(n=14)$, paired normal adjacent tissue $(\mathrm{n}=14)$ and healthy colonic tissue $(n=13)$, were collected for bacterial marker analysis and $16 \mathrm{~S}$ rRNA amplicon sequencing. Microscopic findings were confirmed by an expert gastrointestinal pathologist. All FFPE tissue blocks were processed in neutral-buffered formalin and embedded with paraffin during routine medical practice and obtained from the department of pathology at the Erasmus MC University Medical Center Rotterdam, the Netherlands. In addition, paraffin was sampled from six sources including two batches of paraffin grains, one tissue processor machine and three paraffin embedding stations and transferred into clean autoclaved bottles until bacterial DNA isolation.

\section{Microtome sectioning}

A regular cleaning protocol was applied for processing samples of our 16S rRNA amplicon sequencing pilot, entailing the use of ethanol to clean the microtome and metal tweezers before sectioning. For each block, 14 consecutive sections of $5 \mu \mathrm{M}$ were obtained after disposal of the superficial layers and transferred in autoclaved $1.5 \mathrm{ml}$ Eppendorf tubes for storage until bacterial DNA isolation. A second more stringent contamination-prevention protocol including DNA-Zap treatment of all surfaces and the use of facemasks and flow cabinets was employed to process following specimens (Supplementary methods
1). To control for potential contamination in downstream analysis, paired empty paraffin (0.05 gram) from the same FFPE tissue block was collected using a sterile disposable surgical knife and a clean weighing scale. All specimens were cut within several days of each other, placed in Eppendorf tubes and transferred into a dark box for storage in a cold room to prevent degradation by light/heat until DNA isolation within a few weeks.

\section{Bacterial DNA extraction}

Bacterial DNA isolation of FFPE tissues and paraffin was carried out with the RTP Bacteria DNA Mini Kit (STRATEC Molecular Gm, Berlin, Germany) according to the manufacturer's protocol for FFPE material. The first step was modified using xylene to dissolve paraffin. Melted paraffin $(100 \mu l)$ from six pathology sources served as starting material for DNA isolation with two RTP Bacteria Mini Kits. Autoclaved water $(500 \mu \mathrm{l})$ and/ or blank (no template) samples that were processed by the RTP Bacteria DNA Mini Kit served as additional controls.

For bacterial marker analysis and 16S rRNA amplicon sequencing, FFPE tissue and their paired empty paraffin samples were concurrently processed for DNA extraction in a non-specific order using a stringent decontamination protocol (Supplementary methods 1 ). To prevent repetitive pipetting steps, each DNA sample was divided in aliquots of which one was utilized for 16S rRNA amplicon sequencing. DNA purity was measured with NanoDrop 2000 Spectrophotometer (Thermo Fisher Scientific Inc., Waltham, MA) and concentration with Qubit dsDNA BR Assay Kit (Thermo Fisher). Samples were stored at $-20{ }^{\circ} \mathrm{C}$ until further analysis.

\section{Polymerase chain reaction}

All samples and potential contamination sources, e.g. individual components of three individual RTP Bacteria DNA Mini Kits, extraction additives xylene, ethanol and isopropanol and paraffin were subjected to PCR amplification. PCR assays were executed with an Applied Biosystems 2720 Thermal Cycler (Applied Biosystems, Waltham, MA) using primers targeting the $16 S$ rRNA gene, the human beta-actin (ACTB) gene and Ralstonia species (Supplementary Table S1). Each PCR reaction contained GoTaq ${ }^{\circledR}$ buffer (Promega, Madison, WI), $1.25 \mathrm{mM} \mathrm{MgCl}{ }_{2}$ (Promega), $0.167 \mathrm{mM}$ (each) deoxynucleotides (Roche Diagnostics, Mannheim, Germany), 2.5U GoTaq ${ }^{\circledR}$ polymerase (Promega), 333nM of each primer (Sigma-Aldrich, St Louis, MO), $2 \mu \mathrm{l}$ of template and water to a final volume of $30 \mu \mathrm{l}$. After $4 \mathrm{~min}$ of denaturation at $95^{\circ} \mathrm{C}, 40$ cycles consisting of $30 \mathrm{~s}$ denaturation at $95^{\circ} \mathrm{C}, 30 \mathrm{~s}$ annealing and $1 \mathrm{~min}$ extension at $72{ }^{\circ} \mathrm{C}$ were applied, and followed by the final extension of $10 \mathrm{~min}$ at 
$72{ }^{\circ} \mathrm{C}$. Template was not normalized (undiluted) or normalized to $10 \mathrm{ng} / \mu \mathrm{l}$ where otherwise specified. Water served as negative PCR control and positive controls were fecal bacterial DNA, human genomic DNA from FFPE tissues and known Ralstonia-contaminated elution buffer. Amplicons were visualized by gel electrophoresis using $2 \%$ agarose gel in $1 \mathrm{X}$ TBE (Tris-borate-EDTA) buffer containing Serva DNA stain G (Promega).

\section{Quantitative polymerase chain reaction assays}

Primer details for qPCR assays are described in Supplementary Table S1. To determine bacterial versus human genomic DNA concentration within FFPE tissue samples $(\mathrm{n}=3)$, a standard curve with equimolar Escherichia coli (E.coli) and human genomic DNA was prepared (Supplementary methods 2). The reaction mixture comprised of SYBR Select Master Mix (Applied Biosystems), 200$500 \mathrm{nM}$ of each primer (Sigma-Aldrich), $2 \mu \mathrm{l}$ of normalized template $(10 \mathrm{ng} / \mu \mathrm{l})$ and water for a total volume of $20 \mu \mathrm{l}$ and DNA was amplified using the same cycles as described above. The bacterial versus human genomic DNA concentration were calculated using their respective standard curves and illustrated with Graph Pad Prism 5 software (GraphPad, San Diego, CA). Additionally, $16 \mathrm{~S}$ rRNA gene copy numbers in paired tissue $(n=39)$ and empty paraffin $(n=38)$ samples were calculated and groups analysed with the Wilcoxon test.

Bacterial marker analysis was performed with E.coli and ClbA gene primers to detect E.coli and CRC associated genotoxic strains carrying the pathogenicity island pks [36, 37], respectively. Gut commensal Faecalibacterium prausnitzii (F.prausnitzii), which has been reported to be negatively associated with CRC $[32,33]$, was additionally selected. To account for different FFPE tissue sizes, the $A C T B$ gene was measured. PCR conditions were similar as described above, except for the use of $4 \mu \mathrm{l}$ non-normalized template to enhance amplification. The $2^{-\Delta \Delta C T}$ method was applied to calculate the fold change. The $\Delta \mathrm{CT}_{\text {sample }}\left(=\mathrm{CT}_{\text {bacterial target }}-\mathrm{CT}_{\mathrm{ACTB} \text { target }}\right)$ was first obtained for each sample by normalization to the amount of total human DNA. The average $\Delta \mathrm{CT}_{\text {sample }}$ of the healthy tissues (control group) was then used to calculate $\Delta \Delta \mathrm{CT}\left(=\Delta \mathrm{CT}_{\text {sample }}-\right.$ average $\left.\Delta \mathrm{CT}_{\text {control group }}\right)$, after which the fold change derived from $2^{-\Delta \Delta \mathrm{CT}}$. The KruskalWallis with the Dunn's Multiple Comparison test for post-hoc analysis were performed in Graph Pad Prism 5.

\section{Library preparation and 16S rRNA amplicon sequencing}

$16 \mathrm{~S}$ rRNA amplicon sequencing was performed at the Macrogen Institute, Seoul, Korea, using amplification of the $16 \mathrm{~S}$ rRNA hypervariable region V3-V4 by $341 \mathrm{~F} / 805 \mathrm{R}$ primers. Libraries included paired empty paraffin controls $(n=6)$ and DNA extraction negatives $(n=6)$ for sequencing on the Illumina MiSeq platform $(2 \times 300 \mathrm{bp})$ (Illumina, San Diego, CA). Secondly, a nested PCR approach was applied in house using $27 \mathrm{~F} / 1369 \mathrm{R}$ and $515 \mathrm{~F} / 806 \mathrm{R}$ primers for respectively the initial and a subsequent PCR targeting the V4 region. Paired empty paraffin $(n=41)$, synthetic bacterial mock communities $(n=2)$ and PCR negative controls $(=2)$ were concurrently processed with the tissues. DNA extraction negatives from the aforementioned RTP Bacteria Mini Kits $(n=6)$ were additionally prepared to allow in depth comparison of these controls with the non-nested data set. Sequencing was conducted on the Illumina NovaSeq 6000 platform $(2 \times 150 \mathrm{bp})$ at GATC Biotech (Konstanz, Germany). More details about library preparation and primer sequences are described in Supplementary methods 3 and Supplementary Table S2.

\section{S rRNA amplicon sequencing data processing}

For both the 16S rRNA amplicon sequencing pilot and the two larger data sets (non-nested and nested PCR approaches), quality control of the reads was performed with FASTQC [38] in Java Runtime Environment and Rqc package [39] in $\mathrm{R}$ version 3.5.0 [40]. The NG-Tax pipeline with default settings was applied [41, 42]. The operational taxonomic unit (OTU) table was constructed at $0.1 \%$ abundance threshold, unassigned reads with one mismatch included and chimeras removed. Taxonomic assignment was conducted with the USEARCH algorithm [43] against the Silva SSU 128 database [44]. Further analysis was performed in R with the 'phyloseq' [45], 'microbiome' [46] and 'vegan' [47] packages. Group comparison was conducted based on sample type (tissue, paraffin, controls). Alpha-diversity was computed with Shannon and Chao1 Indices while $\beta$-diversity was assessed with Principal Coordinates Analysis (PCoA) based on weighted UniFrac and Bray-Curtis dissimilarity at genus and OTU level after relative abundance transformation of the data. The 'adonis' permutational multivariate analyses of variance (PERMANOVA) was applied to determine statistical significance between groups. The 'betadisper' function was used to test for multivariate homogeneity of groups dispersons [48]. To ensure reproducibility, the seed was set to 995 for both permutations tests. The biological significance of the data sets was reassessed following removal of contaminants identified by the prevalence method of the 'decontam' package [27]. A 0.5 threshold was set and negative controls consisted of DNA extraction negatives for the non-nested PCR dataset and PCR negatives for the nested PCR approach.

\section{Abbreviations}

ACTB: beta-actin; CRC: colorectal cancer; DNA: deoxyribonucleic acid; FFPE: formalin-fixed paraffin embedded; OTU: operational taxonomic unit; PCOA: 
Principal Coordinates Analysis; PCR: polymerase chain reaction; PERMANOVA: permutational multivariate analyses of variance; $q P C R$ : quantitative polymerase chain reaction; $16 \mathrm{~S}$ rRNA: $16 \mathrm{~S}$ ribosomal RNA.

\section{Supplementary Information}

The online version contains supplementary material available at https://doi. org/10.1186/s12866-021-02359-z.

\section{Additional file 1.}

\section{Acknowledgements}

Not applicable.

\section{Authors' contributions}

SYL, Al, MPP, CB, GMF were involved in the conceptualization and study design. SYL, TV, MD were involved in the sample collection. MD performed the histopathological examination. SYL, Al performed the experiments. Al, PK processed and analysed sequencing data. SYL, Al, TV, PK, CB, GMF were involved in the interpretation and visualisation of the data. PK, CB, MPP, GMF provided supervision. All authors have substantially contributed, were involved in writing, reviewing or editing of the manuscript and approved the final version.

\section{Funding}

Not applicable.

\section{Availability of data and materials}

$16 S$ rRNA amplicon sequences have been deposited in the NCBI SRA database under the BioProject ID PRJNA741803 and PRJNA742764.

\section{Declarations}

Ethics approval and consent to participate

This study has been approved by the ethics committee of the Erasmus University MC Medical Center Rotterdam under the reference number MEC-2018-1301.

\section{Consent for publication}

Not applicable.

\section{Competing interests}

The authors declare that they have no competing interests.

\section{Author details}

${ }^{1}$ Department of Gastroenterology and Hepatology, Erasmus MC University Medical Center Rotterdam, P.O. Box 2040, 3000 CA Rotterdam, The Netherlands. ${ }^{2}$ Laboratory of Microbiology, Wageningen University \& Research, P.O. Box 8033, 6700 EH Wageningen, The Netherlands. ${ }^{3}$ Department of Pathology, Erasmus MC University Medical Center Rotterdam, P.O. Box 2040, 3000 CA Rotterdam, The Netherlands.

Received: 4 April 2021 Accepted: 1 October 2021

Published online: 29 October 2021

\section{References}

1. Kokkat TJ, Patel MS, McGarvey D, LiVolsi VA, Baloch ZW: Archived formalinfixed paraffin-embedded (FFPE) blocks: A valuable underexploited resource for extraction of DNA, RNA, and protein. Biopreserv Biobank 2013, 11(2):101-106.

2. Nagahashi M, Shimada Y, Ichikawa H, Nakagawa S, Sato N, Kaneko K, Homma K, Kawasaki T, Kodama K, Lyle S et al: Formalin-fixed paraffinembedded sample conditions for deep next generation sequencing. J Surg Res 2017, 220:125-132

3. Han HS, Lee KY, Lim SD, Kim WS, Hwang TS: Molecular identification of Helicobacter DNA in human gastric adenocarcinoma tissues using
Helicobacter species-specific 16S rRNA PCR amplification and pyrosequencing analysis. Oncol Lett 2010, 1(3):555-558.

4. Rabelo-Goncalves E, Roesler B, Guardia AC, Milan A, Hara N, Escanhoela C, Almeida J, Boin I, Zeitune JM: Evaluation of five DNA extraction methods for detection of $\mathrm{H}$. pylori in formalin-fixed paraffin-embedded (FFPE) liver tissue from patients with hepatocellular carcinoma. Pathol Res Pract 2014, 210(3):142-146.

5. Lagheden C, Eklund C, Kleppe SN, Unger ER, Dillner J, Sundstrom K: Validation of a standardized extraction method for formalin-fixed paraffinembedded tissue samples. J Clin Virol 2016, 80:36-39.

6. Lynch SV, Pedersen O: The Human Intestinal Microbiome in Health and Disease. N Engl J Med 2016, 375(24):2369-2379.

7. Ursell LK, Metcalf JL, Parfrey LW, Knight R: Defining the human microbiome. Nutr Rev 2012, 70 Suppl 1:S38-44.

8. Bundgaard-Nielsen C, Baandrup UT, Nielsen LP, Sorensen S: The presence of bacteria varies between colorectal adenocarcinomas, precursor lesions and non-malignant tissue. BMC Cancer 2019, 19(1):399.

9. Smith B, Bodé S, Petersen BL, Jensen TK, Pipper C, Kloppenborg J, Boyé M, Krogfelt KA, Mølbak L: Community analysis of bacteria colonizing intestinal tissue of neonates with necrotizing enterocolitis. BMC Microbiol 2011, 11:73-73.

10. Stewart CJ, Fatemizadeh R, Parsons P, Lamb CA, Shady DA, Petrosino JF, Hair AB: Using formalin fixed paraffin embedded tissue to characterize the preterm gut microbiota in necrotising enterocolitis and spontaneous isolated perforation using marginal and diseased tissue. BMC Microbiol 2019, 19(1):52-52.

11. Ullrich T, Tang YW, Correa H, Garzon SA, Maheshwari A, Hill M, Matta P, Krishnan MK, Weitkamp JH: Absence of gastrointestinal pathogens in ileum tissue resected for necrotizing enterocolitis. Pediatr Infect Dis J 2012, 31(4):413-414.

12. Farrugia A, Keyser C, Ludes B: Efficiency evaluation of a DNA extraction and purification protocol on archival formalin-fixed and paraffin-embedded tissue. Forensic Sci Int010, 194(1-3):e25-28.

13. Gilbert MT, Haselkorn T, Bunce M, Sanchez JJ, Lucas SB, Jewell LD, Van Marck E, Worobey M: The isolation of nucleic acids from fixed, paraffinembedded tissues-which methods are useful when? PLoS One 2007, 2(6):e537.

14. Klopfleisch R, Weiss AT, Gruber AD: Excavation of a buried treasure-DNA, mRNA, miRNA and protein analysis in formalin fixed, paraffin embedded tissues. Histol Histopathol 2011, 26(6):797-810.

15. Lin J, Kennedy SH, Svarovsky T, Rogers J, Kemnitz JW, Xu A, Zondervan KT: High-quality genomic DNA extraction from formalin-fixed and paraffinembedded samples deparaffinized using mineral oil. Anal Biochem 2009, 395(2):265-267.

16. Bonin S, Stanta G: Nucleic acid extraction methods from fixed and paraffin-embedded tissues in cancer diagnostics. Expert Rev Mol Diagn 2013, 13(3):271-282.

17. Kotorashvili A, Ramnauth A, Liu C, Lin J, Ye K, Kim R, Hazan R, Rohan T, Fineberg S, Loudig O: Effective DNA/RNA co-extraction for analysis of microRNAs, mRNAs, and genomic DNA from formalin-fixed paraffinembedded specimens. PLoS One 2012, 7(4):e34683.

18. Dedhia P, Tarale S, Dhongde G, Khadapkar R, Das B: Evaluation of DNA extraction methods and real time PCR optimization on formalin-fixed paraffin-embedded tissues. Asian Pac J Cancer Prev 2007, 8(1):55-59.

19. Impraim CC, Saiki RK, Erlich HA, Teplitz RL: Analysis of DNA extracted from formalin-fixed, paraffin-embedded tissues by enzymatic amplification and hybridization with sequence-specific oligonucleotides. Biochem Biophys Res Commun 1987, 142(3):710-716.

20. Gillio-Tos A, De Marco L, Fiano V, Garcia-Bragado F, Dikshit R, Boffetta P, Merletti F: Efficient DNA extraction from 25-year-old paraffin-embedded tissues: study of 365 samples. Pathology 2007, 39(3):345-348.

21. Dahlberg J, Sun L, Persson Waller K, Östensson K, McGuire M, Agenäs S, Dicksved J: Microbiota data from low biomass milk samples is markedly affected by laboratory and reagent contamination. PLoS One 2019, 14(6):e0218257.

22. de Goffau MC, Lager S, Salter SJ, Wagner J, Kronbichler A, Charnock-Jones DS, Peacock SJ, Smith GCS, Parkhill J: Recognizing the reagent microbiome. Nat Microbiol 2018, 3(8):851-853.

23. Glassing A, Dowd SE, Galandiuk S, Davis B, Chiodini RJ: Inherent bacterial DNA contamination of extraction and sequencing reagents may affect 
interpretation of microbiota in low bacterial biomass samples. Gut Pathog 2016, 8:24.

24. Karstens L, Asquith M, Davin S, Fair D, Gregory WT, Wolfe AJ, Braun J, McWeeney S: Controlling for Contaminants in Low-Biomass $16 \mathrm{~S}$ rRNA Gene Sequencing Experiments. mSystems 2019, 4(4):e00290-19.

25. Salter SJ, Cox MJ, Turek EM, Calus ST, Cookson WO, Moffatt MF, Turner P, Parkhill J, Loman NJ, Walker AW: Reagent and laboratory contamination can critically impact sequence-based microbiome analyses. BMC Biol 2014, 12:87.

26. Stinson LF, Keelan JA, Payne MS: Identification and removal of contaminating microbial DNA from PCR reagents: impact on low-biomass microbiome analyses. Lett Appl Microbiol 2019, 68(1):2-8.

27. Davis NM, Proctor DM, Holmes SP, Relman DA, Callahan BJ: Simple statistical identification and removal of contaminant sequences in marker-gene and metagenomics data. Microbiome 2018, 6(1):226.

28. Arumugam M, Raes J, Pelletier E, Le Paslier D, Yamada T, Mende DR, Fernandes GR, Tap J, Bruls T, Batto JM et al: Enterotypes of the human gut microbiome. Nature 2011, 473(7346):174-180.

29. Barton HA, Taylor NM, Lubbers BR, Pemberton AC: DNA extraction from low-biomass carbonate rock: an improved method with reduced contamination and the low-biomass contaminant database. J Microbiol Methods 2006, 66(1):21-31.

30. Erb-Downward JR, Falkowski NR, D'Souza JC, McCloskey LM, McDonald RA, Brown CA, Shedden K, Dickson RP, Freeman CM, Stringer KA et al: Critical Relevance of Stochastic Effects on Low-Bacterial-Biomass 165 rRNA Gene Analysis. mBio 2020, 11(3):e00258-20.

31. Caruso V, Song X, Asquith M, Karstens L: Performance of Microbiome Sequence Inference Methods in Environments with Varying Biomass. mSystems 2019, 4(1):e00163-1.

32. Balamurugan R, Rajendiran E, George S, Samuel GV, Ramakrishna BS: Real-time polymerase chain reaction quantification of specific butyrateproducing bacteria, Desulfovibrio and Enterococcus faecalis in the feces of patients with colorectal cancer. J Gastroenterol Hepatol 2008, 23(8 Pt 1):1298-1303

33. Lopez-Siles M, Martinez-Medina M, Surís-Valls R, Aldeguer X, Sabat-Mir M, Duncan SH, Flint HJ, Garcia-Gil LJ: Changes in the Abundance of Faecalibacterium prausnitzii Phylogroups I and II in the Intestinal Mucosa of Inflammatory Bowel Disease and Patients with Colorectal Cancer. Inflamm Bowel Dis 2016, 22(1):28-41.

34. Pinto-Ribeiro I, Ferreira RM, Pereira-Marques J, Pinto V, Macedo G, Carneiro F, Figueiredo C: Evaluation of the Use of Formalin-Fixed and ParaffinEmbedded Archive Gastric Tissues for Microbiota Characterization Using Next-Generation Sequencing. Int J Mol Sci 2020, 21(3):1096-106.

35. Debesa-Tur G, Pérez-Brocal V, Ruiz-Ruiz S, Castillejo A, Latorre A, Soto JL, Moya A: Metagenomic analysis of formalin-fixed paraffin-embedded tumor and normal mucosa reveals differences in the microbiome of colorectal cancer patients. Sci Rep 2021, 11(1):391.

36. Arthur JC, Perez-Chanona E, Mühlbauer M, Tomkovich S, Uronis JM, Fan TJ, Campbell BJ, Abujamel T, Dogan B, Rogers AB et al: Intestinal inflammation targets cancer-inducing activity of the microbiota. Science 2012, 338(6103):120-123.

37. Pleguezuelos-Manzano C, Puschhof J, Rosendahl Huber A, van Hoeck A Wood HM, Nomburg J, Gurjao C, Manders F, Dalmasso G, Stege PB et al: Mutational signature in colorectal cancer caused by genotoxic pks( $(+)$ E. coli. Nature 2020, 580(7802):269-273.

38. Andrews S: FastQC: a quality control tool for high trhougput sequence data 2010.

39. de Souza W, Carvalho BdS, Lopes-Cendes I: Rqc: A Bioconductor Package for Quality Control of High-Throughput Sequencing Data. 2018, 87(Code Snippet 2):14.

40. R Core Team: R: A language and environment for statistical computing. 2008.

41. Poncheewin W, Hermes GDA, van Dam JCJ, Koehorst JJ, Smidt H, Schaap PJ: NG-Tax 2.0: A Semantic Framework for High-Throughput Amplicon Analysis. Front Genet 2020, 10:1366.

42. Ramiro-Garcia J, Hermes GDA, Giatsis C, Sipkema D, Zoetendal EG, Schaap PJ, Smidt H: NG-Tax, a highly accurate and validated pipeline for analysis of $16 \mathrm{~S}$ rRNA amplicons from complex biomes. F1000Res 2016, 5:1791.

43. Edgar RC: Search and clustering orders of magnitude faster than BLAST. Bioinformatics 2010, 26(19):2460-2461.

44. Quast C, Pruesse E, Yilmaz P, Gerken J, Schweer T, Yarza P, Peplies J, Glockner FO: The SILVA ribosomal RNA gene database project: improved data processing and web-based tools. Nucleic Acids Res 2013, 41(Database issue):D590-596.

45. McMurdie PJ, Holmes S: phyloseq: an R package for reproducible interactive analysis and graphics of microbiome census data. PLoS One 2013, 8(4):e61217.

46. Lahti L, Shetty S: Tools for microbiome analysis in R. Bioconductor 2017.

47. Oksanen J, Blanchet F, Friendly M, Kindt R, Legendre P, McGlinn D, Minchin P, O'Hara R, Simpson G, Solymos P et al: Vegan: community ecology package. 2019.

48. Anderson MJ: Distance-based tests for homogeneity of multivariate dispersions. Biometrics 2006, 62(1):245-253.

\section{Publisher's Note}

Springer Nature remains neutral with regard to jurisdictional claims in published maps and institutional affiliations.
Ready to submit your research? Choose BMC and benefit from:

- fast, convenient online submission

- thorough peer review by experienced researchers in your field

- rapid publication on acceptance

- support for research data, including large and complex data types

- gold Open Access which fosters wider collaboration and increased citations

- maximum visibility for your research: over $100 \mathrm{M}$ website views per year

At BMC, research is always in progress.

Learn more biomedcentral.com/submissions 